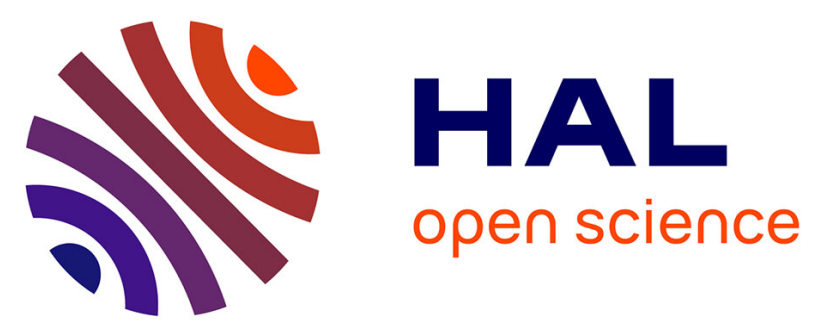

\title{
A Timing Assumption and two t-Resilient Protocols for Implementing an Eventual Leader Service in Asynchronous Shared Memory Systems
}

\author{
Antonio Fernández, Ernesto Jiménez, Michel Raynal, Gilles Trédan
}

\section{To cite this version:}

Antonio Fernández, Ernesto Jiménez, Michel Raynal, Gilles Trédan. A Timing Assumption and two $t$-Resilient Protocols for Implementing an Eventual Leader Service in Asynchronous Shared Memory Systems. [Research Report] PI 1842, 2007, pp.19. inria-00142865

\section{HAL Id: inria-00142865 \\ https://hal.inria.fr/inria-00142865}

Submitted on 23 Apr 2007

HAL is a multi-disciplinary open access archive for the deposit and dissemination of scientific research documents, whether they are published or not. The documents may come from teaching and research institutions in France or abroad, or from public or private research centers.
L'archive ouverte pluridisciplinaire HAL, est destinée au dépôt et à la diffusion de documents scientifiques de niveau recherche, publiés ou non, émanant des établissements d'enseignement et de recherche français ou étrangers, des laboratoires publics ou privés. 


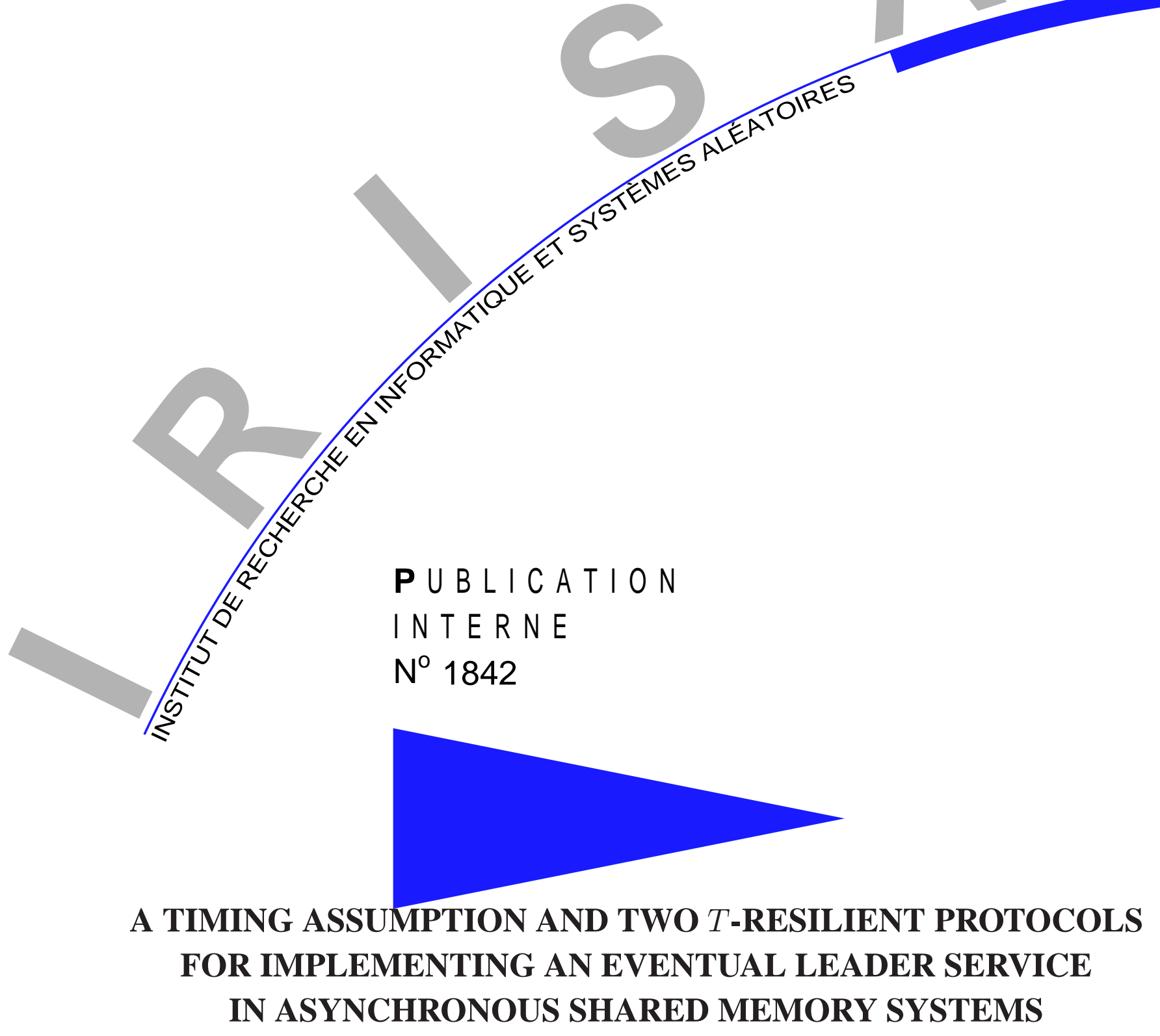
A. FERNÁNDEZ
E. JIMÉNEZ
M. RAYNAL
G. TRÉDAN 



\title{
A Timing Assumption and two $t$-Resilient Protocols for Implementing an Eventual Leader Service in Asynchronous Shared Memory Systems
}

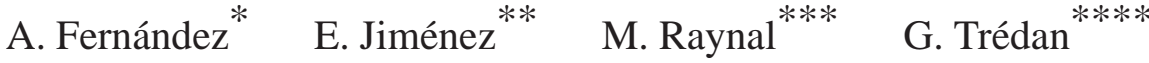 \\ Systèmes communicants \\ Projet ASAP \\ Publication interne $\mathrm{n}^{\circ} 1842$ - Avril 2007 - 19 pages
}

\begin{abstract}
This paper considers the problem of electing an eventual leader in an asynchronous shared memory system. While this problem has received a lot of attention in message-passing systems, very few solutions have been proposed for shared memory systems. As an eventual leader cannot be elected in a pure asynchronous system prone to process crashes, the paper first proposes to enrich the asynchronous system model with an additional assumption. That assumption (denoted $A W B$ ) is particularly weak. It is made up of two complementary parts. More precisely, it requires that, after some time, (1) there is a process whose write accesses to some shared variables be timely, and (2) the timers of $(t-f)$ other processes be asymptotically well-behaved ( $t$ denotes the maximal number of processes that may crash, and $f$ the actual number of process crashes in a run). The asymptotically well-behaved timer notion is a new notion that generalizes and weakens the traditional notion of timers whose durations are required to monotonically increase when the values they are set to increase (a timer works incorrectly when it expires at arbitrary times, i.e., independently of the value it has been set to).

The paper then focuses on the design of $t$-resilient $A W B$-based eventual leader protocols. " $t$-resilient" means that each protocol can cope with up to $t$ process crashes (taking $t=n-1$ provides wait-free protocols, i.e., protocols that can cope with any number of process failures). Two protocols are presented. The first enjoys the following noteworthy properties: after some time only the elected leader has to write the shared memory, and all but one shared variables have a bounded domain, be the execution finite or infinite. This protocol is consequently optimal with respect to the number of processes that have to write the shared memory. The second protocol guarantees that all the shared variables have a bounded domain. This is obtained at the following additional price: all the processes are required to forever write the shared memory. A theorem is proved which states that this price has to be paid by any protocol that elects an eventual leader in a bounded shared memory model. This second protocol is consequently optimal with respect to the number of processes that have to write in such a constrained memory model. In a very interesting way, these protocols show an inherent tradeoff relating the number of processes that have to write the shared memory and the bounded/unbounded attribute of that memory.
\end{abstract}

Key-words: Asynchronous system, Atomic register, Eventual leader, Fault-tolerance, Omega, Process crash, Shared memory, System model, Timer property, Timing assumptions, $t$-resilient protocol, Wait-free protocol.

(Résumé : tsvp)

\footnotetext{
* LADyR, GSyC, Universidad Rey Juan Carlos, 28933 Móstoles, Spain, anto@gsyc . escet . urj c .es

*** EUI, Universidad Politécnica de Madrid, 28031 Madrid, Spain, ernes@eui.upm.es

*** IRISA, Université de Rennes 1, Campus de Beaulieu, 35042, Rennes Cedex, France, raynal@irisa.fr

***** IRISA, Université de Rennes 1, Campus de Beaulieu, 35042, Rennes Cedex, France, gilles.tredan@irisa.fr

The work of A. Fernández and E. Jiménez was partially supported by the Spanish MEC under grants TIN2005-09198-C02-01, TIN2004-07474C02-02, and TIN2004-07474-C02-01, and the Comunidad de Madrid under grant S-0505/TIC/0285. The work of Michel Raynal was supported by the Comunidad de Madrid under grant S-0505/TIC/0285 and the European Network of Excellence ReSIST.
}



Centre National de la Recherche Scientifique (UMR 6074) Université de Rennes 1 - Insa de Rennes

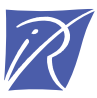

Institut National de Recherche en Informatique et en Automatique - unité de recherche de Rennes 


\section{Election d'un leader dans un système à mémoire partagée asynchrone}

Résumé : Ce rapport présente deux protocoles $t$-resilients d'élection d'un leader inéluctable dans un système réparti défini par des hypothèses de synchronisme très faibles.

Mots clés : Systèmes répartis asynchrones, Tolérance aux fautes, Crash de processus, Oracle oméga, Détection de fautes, Leader inéluctable, Synchronie partielle. 


\section{Introduction}

Context and motivation In order to be able to cope with process failures, many upper layer services (such as atomic broadcast, atomic commitment, group membership, etc.) rely in one form or another on an underlying basic service called eventual leader facility. Such a service provides the processes with a single operation, denoted leader(), such that each invocation of that operation returns a process name, and, after some unknown but finite time, all the invocations returns the same name, and this is the name of an alive process. One of the most famous protocol based on such an eventual leader service is the well-known state machine replication protocol called Paxos [19]. An eventual leader service (also called unreliable failure detector or distributed oracle $[5,29]$ ) is usually denoted $\Omega$ in the literature [6].

Building an eventual leader service requires the processes to cooperate in order to elect one of them. It has been shown that such an election is impossible when the progress of each process is totally independent of the progress of the other processes, namely when the processes are fully asynchronous (direct proofs of this impossibility can be found in $[3,26])$. Of course, considering a synchronous system would allow designing an eventual leader service, but this is not sensible as this is a very strong assumption on the system behavior. So, a central issue consists in finding timing assumptions that are, at the same time, "strong enough" in order a leader service can be built, and "weak enough" in order that they are "practically" meaningful (i.e., they are satisfied nearly always [28]). Finding such necessary and sufficient assumptions remains a fundamental issue from both a practical and theoretical points of view. Seen from a theory point of view, the answer would establish the asynchrony boundary beyond which the problem cannot be solved. Seen from a practical point of view, the answer would define the requirements a system has to satisfy in order to solve the problem, and would consequently provide the engineers with the minimal requirements their underlying systems have to meet.

Some distributed systems are made up of computers that communicate through a network of attached disks. These disks constitute a storage area network (SAN) that implements a shared memory abstraction. As commodity disks are cheaper than computers, such architectures are becoming more and more attractive for achieving fault-tolerance $[1,4,10,21]$. The $\Omega$ protocols presented in this paper are suited to such systems. Examples of shared memory $\Omega$-based protocols can be found in $[9,14]$.

On another side, multi-core architectures are becoming more and more deployed and create a renewed interest for asynchronous shared memory systems. In such a context, it has been shown [11] that $\Omega$ constitutes the weakest contention manager that allows transforming any obstruction-free [16] software transactional memory into a non-blocking transactional memory [17]. This constitutes a very strong motivation to look for requirements that, while being "as weak as possible", are strong enough to allow implementing $\Omega$ in asynchronous shared memory environments prone to process failures.

Content of the paper This paper is on the design of protocols that construct an eventual leader service $\Omega$ in an asynchronous shared memory system where processes can crash. Let $n$ be the total number of processes, and $t$ the maximal number of processes that can crash in a run. We are interested in the design of $t$-resilient protocols, i.e., protocols that can cope with up to $t$ process crashes. This means that the protocol has to work correctly when no more than $t$ processes are faulty. When, more than $t$ processes are faulty, the protocol is allowed to behave arbitrarily. When, $t=n-1$, a $t$-resilient protocol is also called a wait-free protocol [15]. Usually, the system parameter $t$ is explicitly used in the text of a $t$-resilient protocol. As, in practice, the number of processes that crash in a given run is very small, it is interesting to design $t$-resilient protocols. Let $f, 0 \leq f \leq t$, denote the number of processes that crash in a given run. The paper has three main contributions.

CONTRIBUTION \#1. The paper first proposes a behavioral assumption that is particularly weak. The additional assumption the asynchronous system has to satisfy is made up of two matching parts. It is the following. In each run, there are a finite (but unknown) time $\tau$, and a process $p$ that does not crash in that run ( $p$ is not a priori known) such that, after $\tau$ :

- If $f<t$, there is a bound $\Delta$ (not necessarily known) such that any two consecutive write accesses to some shared variables issued by $p$, are separated by at most $\Delta$ time units, and 
- There are $(t-f)$ correct processes $q, q \neq p$, that have a timer that is asymptotically well-behaved. Intuitively, this notion expresses the fact that eventually the duration that elapses before a timer expires has to increase when the timeout parameter increases.

It is important to see that the timers of $n-(t-f)$ correct processes can behave arbitrarily, i.e., they can expire at times that are arbitrary with respect to the values they have been set to. Moreover, the timers of the $(t-f)$ correct processes involved in the additional assumption can behave arbitrarily during arbitrarily long (but finite) periods. Moreover, as we will see in their formal definition, their durations are not required to monotonically increase when their timeout values increase. They only have, after some time, to be lower-bounded by some monotonically increasing function.

It is noteworthy to notice that no process (but $p$ ) is required to have a synchronous behavior, and only some timers have to eventually satisfy a weak behavioral property. Moreover, it is easy to see that, in the runs where $f=t$, the previous assumption is always trivially satisfied despite asynchrony (no process is required to behave synchronously, and no timer is required to behave correctly).

CONTRIBUTION \#2. The paper then presents two $t$-resilient protocols that construct an eventual leader service $\Omega$ in all the runs that satisfy the previous behavioral assumptions. Both protocols use one-writer/multi-readers (1WMR) atomic registers.

- In the first protocol, all the shared registers (but one) have a bounded domain. More specifically, this means that, be the run finite or infinite, there is a time after which only one shared register keeps on increasing. Interestingly, all the timeout values stop increasing.

Moreover, after some time, there is a single process, that writes forever the shared memory. The protocol is consequently write-optimal, as at least one process has to write the shared memory to inform the other processes that the current leader is still alive.

- The second $t$-resilient protocol improves the first one in the sense that all the shared registers used by the processes to communicate are bounded. This nice property is obtained by using two boolean flags and a simple hand-shaking mechanism between each pair of processes. For each ordered pair of processes $(p, q)$, these flags allow, in one direction, $p$ to pass an information to $q$, and in other direction, $q$ to inform $p$ that it has read that information.

Interestingly, the design of both protocols is based on simple ideas. Moreover, these protocols are presented in an incremental way: the second $t$-resilient protocol is designed as a simple improvement of the first one. This make easier both its understanding and its proof.

CONTRIBUTION \#3. The paper proves lower bound results for the considered computing model. These results concern the minimal number of processes that have to write the shared memory when that memory is not bounded and when it is bounded, and the minimal number of processes that have to read the shared memory.

More precisely, three theorems are stated and proved. The first shows that the process that is eventually elected has to forever write the shared memory. Another theorem shows that any process (but the eventual leader) has to forever read the shared memory. Finally, the last theorem shows that, if the shared memory is bounded, then all the processes have to forever write the shared memory. These theorems show that the two $t$-resilient protocols presented in the paper are optimal with respect to these criteria.

Related work in the message-passing context The design of protocols that implements an eventual leader service has received a lot of attention in the message-passing context, i.e., when the processes cooperate by exchanging messages through an underlying network. The implementation of $\Omega$ in asynchronous message-passing systems is an active research area. Two main approaches have been investigated: the timer-based approach and the message pattern-based approach.

The timer-based approach relies on the addition of timing assumptions [7]. Basically, it assumes that there are bounds on process speeds and message transfer delays, but these bounds are not known and hold only after some finite but unknown time. The protocols implementing $\Omega$ in such "augmented" asynchronous systems are based on timeouts (e.g., $[2,3,20])$. They use successive approximations to eventually provide each process with an upper bound on transfer delays and processing speed. They differ mainly on the "quantity" of additional synchrony they consider, and on the message cost they require after a leader has been elected. 
Among the protocols based on this approach, a protocol presented in [2] is particularly attractive, as it considers a relatively weak additional synchrony requirement. Let $t$ be an upper bound on the number of processes that may crash ( $1 \leq t<n$, where $n$ is the total number of processes). This assumption is the following: the underlying asynchronous system, which can have fair lossy channels, is required to have a correct process $p$ that is a $\diamond t$-source. This means that $p$ has $t$ output channels that are eventually timely: there is a time after which the transfer delays of all the messages sent on such a channel are bounded (let us notice that this is trivially satisfied if the receiver has crashed). Notice that such a $\diamond t$-source is not known in advance and may never be explicitly known. It is also shown in [2] that there is no leader protocol if the system has only $\diamond(t-1)$-sources. A versatile adaptive timer-based approach has been developed in [23].

The message pattern-based approach, introduced in [24], does not assume eventual bounds on process and communication delays. It considers that there is a correct process $p$ and a set $Q$ of $t$ processes (with $p \notin Q$, moreover $Q$ can contain crashed processes) such that, each time a process $q \in Q$ broadcasts a query, it receives a response from $p$ among the first $(n-t)$ corresponding responses (such a response is called a winning response). It is easy to see that this assumption does not prevent message delays to always increase without bound. Hence, it is incomparable with the synchrony-related $\diamond t$-source assumption. This approach has been applied to the construction of an $\Omega$ protocol in [26].

A hybrid protocol that combines both types of assumption is developed in [27]. More precisely, this protocol considers that each channel eventually is timely or satisfies the message pattern, without knowing in advance which assumption it will satisfy during a particular run. The aim of this approach is to increase the assumption coverage, thereby improving fault-tolerance [28].

Related work in the shared memory context To our knowledge, only three eventual leader protocols suited to the shared memory context has been proposed so far $[8,13]$. The protocol presented in [13] assumes that there is a finite time after which all the processes behave synchronously. So, this timing assumption is pretty strong.

The second paper [8] investigates a weaker assumption that is close to the assumption defined here. The main difference lies in the fact that the assumption proposed in [8] works only for $t=n-1$, which means that it considers only the wait-free case and the corresponding protocols are not $t$-resilient for $1 \leq t<n-1$. The current paper can be seen as a generalization of the results of [8] to obtain $t$-resilient protocols.

Roadmap The paper is made up of 5 sections. Section 2 presents the system model and the additional behavioral assumption. Then, Sections 3 and 4 present in an incremental way the two $t$-resilient protocols implementing an eventual leader service, and show they are optimal with respect to the number of processes that have to write or read the shared memory. Finally, Section 5 provides concluding remarks.

\section{System model, eventual leader, and additional assumption}

\subsection{Base asynchronous shared memory model}

The system consists of $n(n>1)$ processes denoted $p_{1}, \ldots, p_{n}$. We assume that process identities are all different and totally ordered. Hence, for simplicity we make the integer $i$ to denote the identity of $p_{i}$. A process can fail by crashing, i.e., prematurely halting. Until it possibly crashes, a process behaves according to its specification, namely, it executes a sequence of steps as defined by its protocol. After it has crashed, a process executes no more steps. By definition, a process is faulty during a run if it crashes during that run; otherwise it is correct in that run. In the following, $t$ denotes the maximum number of processes that are allowed to crash in any run $(1 \leq t \leq n-1)^{1}$, while $f$ denotes the actual number of processes that crash in a run $(0 \leq f \leq t)$.

The processes communicate by reading and writing a memory made up of atomic registers (also called shared variables). Each register is one-writer/multi-reader (1WMR). "1WMR" means that a single process can write into it, but all the processes can read it. (Let us observe that using 1WMR atomic registers is particularly suited for cached-

${ }^{1}$ This means that, if more than $t$ processes crash in a run, we are outside the system model, and a protocol can then behave arbitrarily. If we want the protocol to cope with any number of process crashes we have to take $t=n-1$. 
based distributed shared memory. $)^{2}$ The only process allowed to write an atomic register is called its owner. Atomic means that, although read and write operations on the same register may overlap, each (read or write) operation appears to take effect instantaneously at some point of the time line between its invocation and return events (this is called the linearization point of the operation [18]). Uppercase letters are used for the identifiers of the shared registers. These registers are structured into arrays. As an example, PROGRESS $[i]$ denotes a shared register that can be written only by $p_{i}$, and read by any process. A process can have local variables. Those are denoted with lowercase letters, with the process identity appearing as a subscript. As an example, progress $_{i}$ denotes a local variable of $p_{i}$.

Some shared registers are critical, while other shared registers are not. A critical register is an atomic register on which some constraint can be imposed by the additional assumptions that allow implementing an eventual leader. This attribute allows restricting the set of registers involved in these assumptions.

This base model is characterized by the fact that there is no assumption on the execution speed of one process with respect to another. This is the classical asynchronous shared memory model where up to $t$ processes may crash. It is denoted $\mathcal{A S}_{n, t}[\emptyset]$ in the following.

\subsection{Eventual leader service}

The notion of eventual leader service has been informally presented in the introduction. It is an entity that provides each process with a primitive leader() that returns a process identity each time it is invoked. A unique correct leader is eventually elected but there is no knowledge of when the leader is elected. Several leaders can coexist during an arbitrarily long period of time, and there is no way for the processes to learn when this "anarchy" period is over. The leader service, denoted $\Omega$, satisfies the following properties [6]. (The second property refers to a notion of global time. It is important to notice that this global time is only for a specification purpose. It is not accessible to the processes.)

- Validity: The value returned by a leader() invocation is a process identity.

- Eventual Leadership: There is a finite time and a correct process $p_{i}$ such that, after that time, every leader() invocation returns $i$.

- Termination: Any leader() invocation issued by a correct process terminates.

The $\Omega$ leader abstraction has been formally introduced in [6]. It has been shown to be weakest, in terms of information about failures, to solve consensus in asynchronous systems prone to process crashes, be these systems message-passing systems [6] or shared memory systems [22]. Several consensus protocols based on such an eventual leader service have been proposed (e.g., [12, 19, 25] for message-passing systems, and [9, 14] for shared memory systems).

\subsection{Additional behavioral assumption}

Underlying intuition As already indicated, $\Omega$ cannot be implemented in pure asynchronous systems such as $\mathcal{A S}_{n, t}[\emptyset]$. So, we consider the system is no longer fully asynchronous: its runs satisfy the following assumption denoted $A W B$ (for asymptotically well-behaved). The resulting system is consequently denoted $\mathcal{A S}_{n, t}[A W B]$.

Each process $p_{i}$ is equipped with a timer denoted timer $_{i}$. The intuition that underlies $A W B$ is that, once a process $p_{\ell}$ that has not crashed is defined as being the current leader, it should not to be demoted by a process $p_{i}$ that believes $p_{\ell}$ has crashed. To that end, constraints have to be defined on the behavior of both $p_{\ell}$ and $p_{i}$. The constraint on $p_{\ell}$ is to force it to "regularly" inform the other processes that it is still alive. The constraint on a process $p_{i}$ is to prevent it to falsely suspect that $p_{\ell}$ has crashed.

There are several ways to define runs satisfying the previous constraints. As an example, restricting the runs to be "eventually synchronous" [5, 7] would work but is much more constraining than what is necessary. The aim of the $A W B$ additional assumption is to state constraints that are "as weak as possible" ${ }^{3}$. It appears that requiring the timers to be eventually monotonous is stronger than necessary (as we are about to see, this is a particular case of the $A W B$

\footnotetext{
${ }^{2}$ As observed in the Introduction the atomic registers can also be seen as a high level abstraction of a communication system made up of commodity disks. Such disks can be accessed only by read and write operations. Such "shared memory" systems are described in [10, 21]. Protocols based of commodity disks are described in $[9,14]$.

${ }^{3}$ Of course, the notion of "as weak as possible" has to be taken with its intuitive meaning. This means that, when we want to implement $\Omega$ in a shared memory system, we know neither an assumption weaker than $A W B$, nor the answer to the question: Is $A W B$ the weakest additional assumption?
} 
assumption). The $A W B$ assumption is made up of two parts $A W B_{1}$ and $A W B_{2}$ that we present now. $A W B_{1}$ is on the existence of a process whose behavior has to satisfy a synchrony property. $A W B_{2}$ is on the timers of other processes. $A W B_{1}$ and $A W B_{2}$ are "matching" properties.

The assumption $A W B_{1}$ That assumption restricts the asynchronous behavior of one process. Given a run characterized by a value of $f$, it is defined as follows.

$A W B_{1}$ : If $f<t$, there are a time $\tau_{A W B_{1}}$, a bound $\Delta$, and a correct process $p_{\ell}\left(\tau_{A W B_{1}}, \Delta\right.$ and $p_{\ell}$ may never be explicitly known) such that, after $\tau_{A W B_{1}}$, any two consecutive write accesses issued by $p_{\ell}$ to (its own) critical registers, are completed in at most $\Delta$ time units.

Let us first observe that this assumption is always satisfied when $f=t$. When $f<t$, it means that, after some arbitrary (but finite) time, the speed of $p_{\ell}$ is lower-bounded, i.e., its behavior is partially synchronous (let us notice that, while there is a lower bound, no upper bound is required on the speed of $p_{\ell}$, except the fact that it is not $+\infty$ ). In the following we say " $p_{\ell}$ satisfies $A W B_{1}$ " to say that $p_{\ell}$ is a process that makes true that assumption.

The assumption $A W B_{2}$ The definition of $A W B_{2}$ involves timers and relies on the notion of asymptotically wellbehaved timer. The aim of that notion is to capture timer behaviors that are sufficient to implement an eventual leader but could be too weak to solve other problems. From an operational point of view, the intuition that underlies that notion is that there is a time $\tau$ after which, whatever the duration $\delta$ and the time $\tau^{\prime} \geq \tau$ at which it is set to $\delta$, that timer expires after some finite time $\tau^{\prime \prime}$ such that $\tau^{\prime \prime} \geq \tau^{\prime}+\delta$. That is the only constraint on the timer expiration for that timer to be asymptotically well-behaved. If the timer is set to $\delta 1$ at some time $\tau 1 \geq \tau$ and expires at $\tau 1^{\prime}$, and the same or another timer is set to $\delta 2>\delta 1$ at some time $\tau 2 \geq \tau$ and expires at $\tau 2^{\prime}$, it is not required that $\tau 2^{\prime}-\tau 2>\tau 1^{\prime}-\tau 1$.

In order to formally define the notion of asymptotically well-behaved timer, we first introduce a function $f()$ with monotonicity properties that will be used to define an asymptotic behavior. That function takes two parameters, a time $\tau$ and a duration $x$, and returns a duration. It is defined as follows. There are two (possibly unknown) bounded values $x_{A W B_{2}}$ and $\tau_{A W B_{2}}$ such that:

- (f1) $\forall \tau_{2}, \tau_{1}: \tau_{2} \geq \tau_{1} \geq \tau_{A W B_{2}}, \forall x_{2}, x_{1}: x_{2} \geq x_{1} \geq x_{A W B_{2}}: f\left(\tau_{2}, x_{2}\right) \geq f\left(\tau_{1}, x_{1}\right)$. (After some point, $f($ ) is not decreasing with respect to $\tau$ and $x$ ).

- (f2) $\lim _{x \rightarrow+\infty} f\left(\tau_{A W B_{2}}, x\right)=+\infty$. (Eventually, $f()$ always increases ${ }^{4}$.)

Thanks to the function $f()$, we are now in order to give a general and precise definition for the notion of asymptotically well-behaved timer. Considering the timer timer $_{i}$ of a process $p_{i}$ and a run $R$, let $\tau$ be a real time at which the timer is set to a value $x$, and $\tau^{\prime}$ be the finite real time at which that timer expires. Let $T_{R}(\tau, x)=\tau^{\prime}-\tau$, for each $x$ and $\tau$. Then timer timer $_{i}$ is asymptotically well-behaved in a run $R$, if there is a function $f_{R}()$, as defined above, such that:

- (f3) $\forall \tau: \tau \geq \tau_{A W B_{2}}, \forall x: x \geq x_{A W B_{2}}: f_{R}(\tau, x) \leq T_{R}(\tau, x)$.

This constraint states the fact that, after some point, the function $T_{R}()$ is always above the function $f_{R}()$. It is important to observe that, after $\left(\tau_{A W B_{2}}, x_{A W B_{2}}\right)$, the function $T_{R}(\tau, x)$ is not required to be non-decreasing, it can increase and decrease. Its only requirement is to always dominate $f_{R}()$. (See Figure 1.)

An asymptotically well-behaved timer is allowed to expire at arbitrary times (i.e., times that are unrelated to the timeout values it has been set to) during an arbitrary but finite time, after which it behaves correctly in the sense that it never expires "too early". There is no upper bound on the duration after which it expires, except that this duration is finite.

The assumption $A W B_{2}$ can now be stated. It is the following.

$A W B_{2}$ : The timers of $(t-f)$ correct processes (different from the process $p_{\ell}$ that satisfies $A W B_{1}$ ) are asymptotically well-behaved.

\footnotetext{
${ }^{4}$ If the image of $f()$ is the set of natural numbers, then this condition can be replaced by $x_{2}>x_{1} \Longrightarrow f\left(\tau_{A W B_{2}}, x_{2}\right)>f\left(\tau_{A W B_{2}}, x_{1}\right)$.
} 


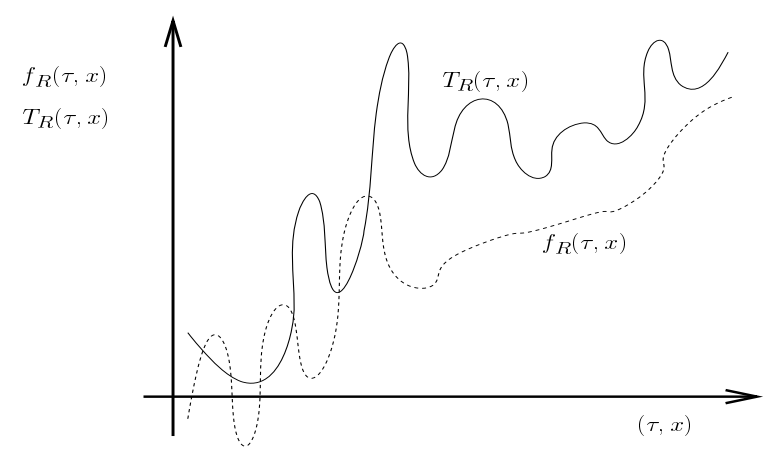

Figure 1: $T_{R}()$ asymptotically dominates $f_{R}()$

When we consider $A W B$, it is important to notice that any process (but $p_{\ell}$ constrained by a speed lower bound) can behave in a fully asynchronous way. Moreover, the local clocks used to implement the timers are required to be neither synchronized, nor accurate with respect to real-time. Moreover, the timers of up to $(n-t)+f$ correct processes can behave arbitrarily. This means that, in the runs where $f=t$, the timers can behave arbitrarily. It follows that the timing assumption $A W B$ is particularly weak.

In the following we say " $p_{x}$ is involved in $A W B_{2}$ " to say that $p_{x}$ is a correct process that has an asymptotically well-behaved timer.

\section{A write-optimal $t$-resilient protocol for $\mathcal{A} \mathcal{S}_{n, t}[A W B]$}

\subsection{Principle of the protocol}

The first $t$-resilient protocol that implements an eventual leader in $\mathcal{A S}_{n, t}[A W B]$ is described in Figure 2. It is based on a simple idea: a process $p_{i}$ elects the process that is the least suspected to have crashed (that idea is used in a lot of eventual leader election protocols in message-passing systems). So, each time a process $p_{i}$ suspects its current leader $p_{j}$ because it has not observed a progress from $p_{j}$ during some duration (defined by the latest timeout value used to set its timer), it increases a suspicion counter (denoted SUSPICIONS $[i, j]$ ).

It is possible that, because its timer does not behave correctly, a process $p_{i}$ suspects erroneously a process $p_{k}$, despite the fact that $p_{k}$ did some progress (this progress being made visible thanks to assumption $A W B_{1}$ if $p_{k}$ satisfies that assumption). So, when it has to determine its current leader, $p_{i}$ does not consider the whole set of suspicions (the array SUSPICIONS[1..n, 1..n]), but only an appropriate part of it. More precisely, for each process $p_{k}, p_{i}$ takes into account only the $(t+1)$ entries with the smallest values among the $n$ counters $S U S P I C I O N S[1, k], \ldots, S U S P I C I O N S[n, k]$. As we will see, due $A W B_{2}$, this allows it to eliminate the erroneous suspicions and consequently determine a correct eventual common leader.

As several processes can be equally suspected, $p_{i}$ uses the function lex_min $(X)$ that outputs the lexicographically smallest pair in the set parameter $X$, where $X$ is a set of (number of suspicions, process identity) pairs and $(a, i)<$ $(b, j)$ iff $(a<b) \vee(a=b \wedge i<j)$.

\subsection{Shared and local variables}

Shared variables The shared memory is made up of a size $n$ vector plus a $n \times n$ matrix of $1 \mathrm{WMR}$ shared atomic registers.

- PROGRESS $[1 . . n]$ is an array of $1 \mathrm{WMR}$ shared integer variables. Only $p_{i}$ can write PROGRESS $\left.i\right]$. In order to indicate to the other processes that it is still alive, $p_{i}$ regularly increases PROGRESS $\left.i i\right]$ when it considers it is the leader.

- SUSPICIONS[1..n, 1..n] is an array of shared variables that contain non-negative integers. The entries of the vector $S U S P I C I O N S[i, 1 . . n]$ can be written only by $p_{i}$. Intuitively, $S U S P I C I O N S[i, j]=x$ means that, up to now, the process $p_{i}$ has suspected $x-1$ times the process $p_{j}$ to have crashed. 
Each shared variable PROGRESS $[k], 1 \leq k \leq n$, is critical. Differently, none of the shared variables $S U S P I C I O N S[k, \ell]$, $1 \leq k, \ell \leq n$, is critical. This means that, for a process $p_{k}$ involved in the assumption $A W B_{1}$, only the write accesses to PROGRESS $[k]$ are concerned.

To achieve correctness, the initial values of the previous shared variables could be arbitrary ${ }^{5}$. However, to make the presentation easier, improve efficiency, and reach optimality in some cases, we consider in the following that initially SUSPICIONS $[i, j]=1,1 \leq i, j \leq n, i \neq j$, and SUSPICIONS $[i, i]=0,1 \leq i \leq n$.

Local variables Each process $p_{i}$ manages the following local variables.

- progress $_{i}$ is used by $p_{i}$ to measure its progress, and consequently update PROGRESS[i].

- last $_{i}[1 . . n]$ is an array such that $l_{a s t_{i}}[k]$ contains the last value of PROGRESS $[k]$ read by $p_{i}$.

- suspicions $s_{i}[1 . . n]$ is an array such that suspicions $_{i}[k]$ contains the number of times $p_{i}$ suspected $p_{k} ;$ suspicions $_{i}[k]$ is used to update $S U S P I C I O N S[i, k]$.

- timeout $_{i}$ contains the last timeout value used by $p_{i}$ to set its timer timer $_{i}$.

- susp_count $t_{i}$ is a variable used to count the current number of meaningful suspicions of $p_{i}$ (issued by the other processes); prev_susp_count ${ }_{i}$ is used to keep the previous value of susp_count ${ }_{i}$.

- progress $_{\_} k_{i}$, witness_k $k_{i}$, my_witnesses $_{i}$, witness $s_{i}[1 . . n]$ and $\operatorname{susp}_{i}[1 . . n]$ are auxiliary local variables used by $p_{i}$.

\subsection{Process behavior}

The behavior of a process $p_{i}$ is described in Figure 2. It is decomposed in three tasks.

Task $T 1$ The first task (lines 1-6) defines the way the current leader is determined. For each process $p_{k}, p_{i}$ first computes the number of relevant suspicions that concern $p_{k}$. As already mentioned, those are defined by the $(t+1)$ entries of the vector SUSPICIONS[1..n, $k]$ with the smallest values (lines 3-4). The $(t+1)$ processes whose entries in SUSPICIONS[1..n, $k]$ have the smallest values are called the witness processes for $p_{k}$. The current leader is then defined as the process that is currently the least suspected, when considering only the relevant suspicions, i.e., for each process $p_{k}$, the suspicions issued by its witness processes (line 6).

Task T2 The second task (lines 7-14) is an infinite loop that is on the management of the shared variable PROGRESS $[i]$ (line 11). More explicitly, a process $p_{i}$ increases PROGRESS $\left.i\right]$ when it considers that it is the leader (test leader ()$=$ $i$, line 11), or when the number of its relevant suspicions has changed since the last time it has executed that task (test susp_count $_{i} \neq$ prev_susp_count $_{i}$, line 11 ; this means that $p_{i}$ has been considered as a leader since its last execution of $T 2$ ). This allows $p_{i}$ to inform the processes that suspected it that it is still alive.

Task $T 3$ The third task is associated with $p_{i}$ 's timer expiration. It is where $p_{i}$ possibly suspects the current leader and where it sets its timer $\left(\right.$ timer $\left._{i}\right)$.

1. Suspicion management part (lines 16-27). First, $p_{i}$ determines its current leader $p_{k}$ (line 16) and the current set of $(t+1)$ processes that suspect the least $p_{k}$; these processes define the set witness ${ }_{i}[k]$ (line 17). A process $p_{i}$ is allowed to worry about its current leader $p_{k}$ (line 19) only if (1) it does not consider itself as the current leader (i.e., $i \neq k$ ), (2) it belongs to the set of $p_{k}$ 's witnesses ( $i \in$ witness_ $k_{i}$ ), and, (3) at the previous timer expiration, $p_{k}$ was its leader $\left(k=\right.$ prev $\left.l d_{i}\right)$ and, since that time, $p_{k}$ has not seen an increase in its number of relevant suspicions $\left(s u s p \_k_{i}=\right.$ prev $\left.\_s u s p_{i}\right)$. The predicate $\left(k=\right.$ prev $\left.l d_{i}\right) \wedge\left(s u s p \_k_{i}=\right.$ prev_susp $\left.p_{i}\right)$ allows $p_{i}$ for checking that $p_{k}$ was continuously the leader between two consecutive expirations of timer $_{i}$. So,

\footnotetext{
${ }^{5}$ This means that the protocol is self-stabilizing with respect to the shared variables. Whatever their initial values, it converges in a finite number of steps towards a common leader, as soon as the additional assumption is satisfied. When these variables have arbitrary initial values (that can be negative), the statement "set timer $_{i}$ to timeout $_{i}$ " (line 28 of Figure 2 ) has to be replaced by "set timer $_{i}$ to $\max \left(\right.$ timeout $\left._{i}, 1\right)$ " in order a timer be always set to a positive value.

PI ${ }^{\circ} 1842$
} 


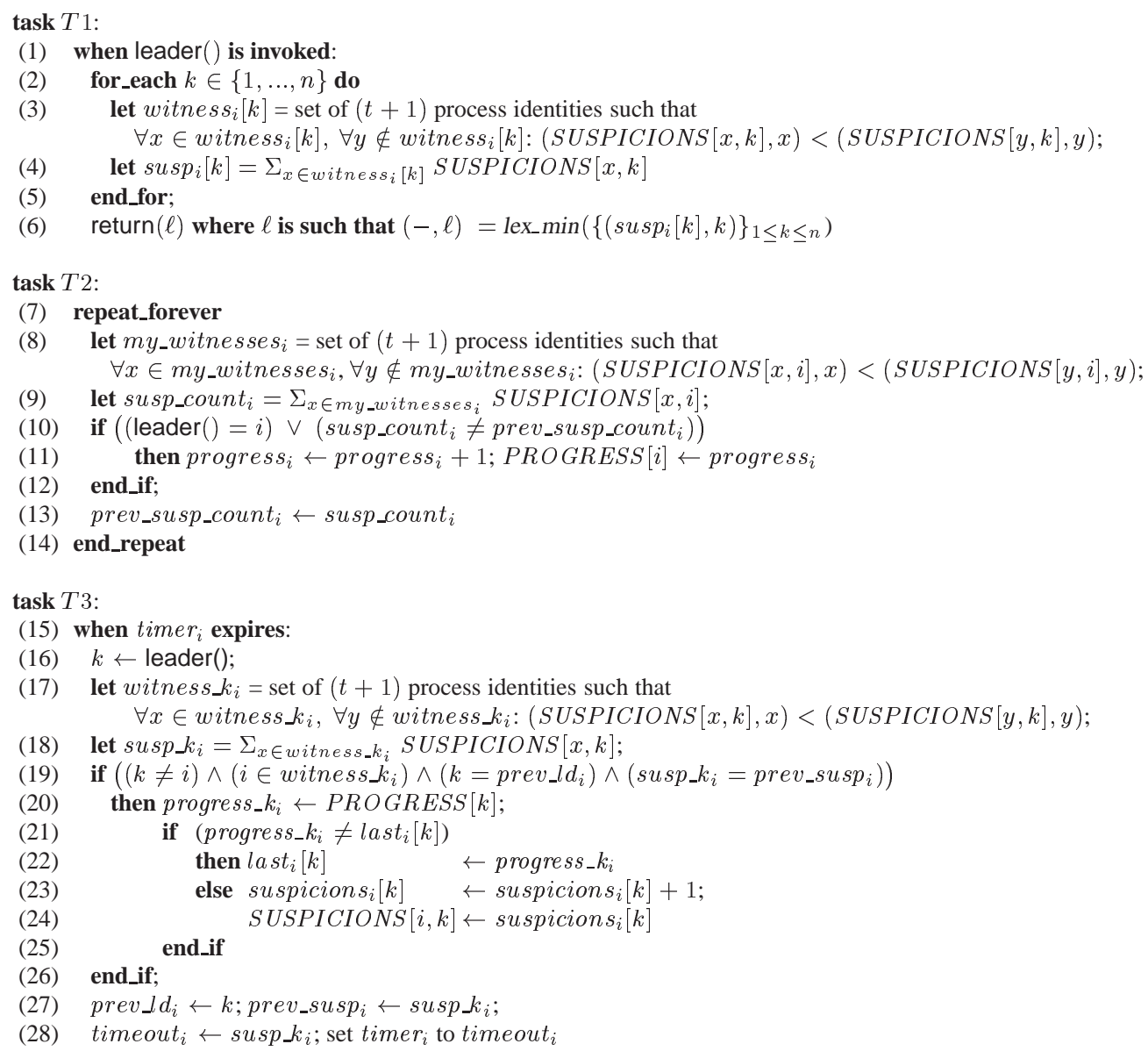

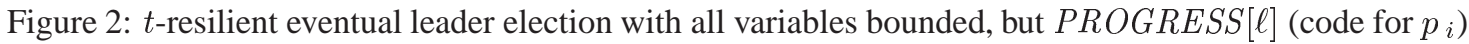

when the the predicate of line 19 is satisfied, $p_{i}$ reads the value of PROGRESS[k] (line 20) to see if it has been increased since its last reading (line 21). If it is the case, $p_{i}$ updates $l a s t_{i}[k]$ accordingly (line 22). If it is not the case, $p_{i}$ suspects once more $p_{k}$ (line 24). As we can see, in order to check if its leader $p_{k}$ is alive or in order to suspect it, a process $p_{i}$ has to currently be one of the witness of $p_{k}$. Finally, $p_{i}$ updates the values of prev $l d_{i}$ and prev_susp $_{i}$ (line 27).

2. Timer setting part (line 28). Then, $p_{i}$ resets its timer to an appropriate timeout value. That value is the number of current relevant suspicions that has been computed in $\operatorname{susp} k_{i}$. Let us observe that, if the leader does not change and the number of its relevant suspicions does no longer increase, timeout ${ }_{i}$ keeps forever the same value.

\subsection{Proof of the protocol}

Let us consider a run $R$ of the protocol described in Figure 2 in which the assumptions $A W B_{1}$ and $A W B_{2}$ defined in Section 2.3 are satisfied. This section shows that an eventual leader is elected in that run. The proof is decomposed into several lemmas.

Lemma 1 Let $p_{i}$ be a faulty process. For any $p_{j}$, SUSPICIONS $[i, j]$ is bounded.

Proof Let us first observe that the vector SUSPICIONS $[i, 1 . . n]$ is updated only by $p_{i}$. The proof follows immediately from the fact that, after it has crashed, a process does no longer modify shared variables. 
Lemma 2 Assuming $f<t$, let $p_{i}$ be a correct process involved in $A W B_{2}$ (i.e., its timer is eventually well-behaved), and $p_{j}$ a correct process that satisfies $A W B_{1}$. Then, SUSPICIONS $[i, j]$ is bounded.

Proof Let $S$ be the sequence of updates of PROGRESS[j] issued by $p_{j}$. Let us observe that all these updates are issued by the task $T 2$ of $p_{j}$ (line 11). We consider two cases.

- $S$ is finite ${ }^{6}$.

In that case, there is a finite time $\tau$ after which the predicate $(\operatorname{leader}() \neq j) \wedge\left(\right.$ susp_count $_{j}=$ prev_susp_count $\left._{j}\right)$ evaluated by $p_{j}$ (line 10) is always true. It follows from this observation and line 13 that, after $\tau$, the local predicate susp_count $_{j}=$ prev_susp_count $_{j}$ remains permanently true.

Assume now, by way of contradiction, that $S U S P I C I O N S[i, j]$ never stops increasing. Then, from the above local predicate, there is a time $\tau^{\prime}$ after which process $p_{i}$ is never among the $(t+1)$ witnesses of $p_{j}$. (These witness processes are defined at line 17.) Note that the condition at line 19 forces that in order to increase SUSPICIONS $[x, j]$ (line 24), a process $p_{x}$ has to consider itself as one of the $(t+1)$ witnesses of $p_{j}$. We conclude that, after $\tau^{\prime}, S U S P I C I O N S[i, j]$ is never increased.

- $S$ is infinite.

Due to the assumption $A W B_{1}$, there are a time $\tau_{A W B_{1}}$, and a bound $\Delta$ such that, after $\tau_{A W B_{1}}$, any two consecutive updates of PROGRESS $[j]$ by $p_{j}$ are completed by at most $\Delta$ time units.

By assumption $A W B_{2}$, the timer of $p_{i}$ is asymptotically well-behaved, which means that, for each run $R$, there are a function $f_{R}()$ and parameters $\tau_{A W B_{2}}$ and $x_{A W B_{2}}$. Let $x_{0} \geq x_{A W B_{2}}$ be a finite value such that $f_{R}\left(\tau_{A W B_{2}}, x_{0}\right)=\Delta^{\prime}>\Delta$. Assumption (f2) implies that such a value $x_{0}$ does exist.

All the time instants considered in the following are after $\max \left(\tau_{A W B_{1}}, \tau_{A W B_{2}}\right)$. Let us assume (by contradiction) that $S U S P I C I O N S[i, j]$ increases forever.

1. As $S U S P I C I O N S[i, j]$ increases forever (line 24 ), it follows that $p_{i}$ is a witness of $p_{j}$ infinitely often (test of line 19), which means that SUSPICIONS $[i, j]$ is infinitely often one of the $(t+1)$ smallest value in the vector SUSPICIONS[1..n, $j]$. We conclude that there is a time $\tau^{\prime}$ after which $s u s p \_k_{i}>x_{0}$ (lines 17 and 18). Consequently, after $\tau^{\prime}$, any two successive expirations of timer $_{i}$ are separated by at least $\Delta^{\prime}$ time units (line 28).

2. As, just before $S U S P I C I O N S[i, j]$ is increased, the predicate $(j=$ prev $\left.\lrcorner d_{i}\right) \wedge\left(s u s p \_k_{i}=\right.$ prev $\left.\_s u s p \_d_{i}\right)$ is true (test of line 19), it follows that, during at least $\Delta^{\prime}$ time units, $p_{j}$ has been the leader without being demoted. (The fact that $p_{j}$ has not been demoted follows from the following observation. As the SUSPICIONS $[x, y]$ variables can only increase, we can conclude from susp $k_{i}=$ prev_susp $\_l d_{i}$ that the number of relevant suspicions of $p_{j}$ have not increased and consequently $p_{j}$ has been continuously the leader between the end of the first computation of susp $k_{i}$-kept in prev_susp $\lrcorner d_{i}$ - and the beginning of the following computation of susp $\rfloor_{i}$.)

As $\Delta^{\prime}>\Delta, A W B_{1}$ is satisfied by $p_{j}$, and we are after $\tau_{A W B_{1}}$, it follows that $p_{j}$ has increased its critical variable PROGRESS $[j]$ between the any two successive readings of that variable by $p_{i}$. It follows that necessarily we then have $l_{a s t}[j] \neq P R O G R E S S[j]$, and the test of line 21 is consequently satisfied. It follows that, after $\max \left(\tau_{A W B_{1}}, \tau_{A W B_{2}}, \tau^{\prime}\right)$, the variable $S U S P I C I O N S[i, j]$ can no longer be increased, contradicting the assumption that it increases forever. This completes the proof of the lemma. $\square_{\text {Lemma } 2}$

Notation 1 Given a process $p_{k}$, let $s k_{1}(\tau) \leq s k_{2}(\tau) \leq \cdots \leq s k_{t+1}(\tau)$ denote the $(t+1)$ smallest values among the $n$ values in the vector SUSPICIONS $[1 . . n, k]$ at time $\tau$ (i.e., these values are the number of suspicions issued by the processes that are the witnesses of $p_{k}$ at time $\left.\tau\right)$. Let $M_{k}(\tau)$ denote $s k_{1}(\tau)+s k_{2}(\tau)+\cdots+s k_{t+1}(\tau)$.

Notation 2 Let $S$ denote the set containing the $f$ faulty processes plus the $(t-f)$ correct processes involved in the assumption $A W B_{2}$ (their timers are asymptotically well-behaved). Then, for each process $p_{k} \notin S$, let $S_{k}$ denote the set $S \cup\left\{p_{k}\right\}$. (Let us notice that $\left|S_{k}\right|=t+1$.)

\footnotetext{
${ }^{6}$ In that case, the fact that $p_{i}$ satisfies $A W B_{2}$ and $p_{j}$ satisfies $A W B_{1}$, is irrelevant.
} 
Lemma 3 At any time $\tau$, there is a process $p_{i} \in S_{k}$ such that the predicate SUSPICIONS $[i, k] \geq s k_{t+1}(\tau)$ is satisfied.

Proof Let $K(\tau)$ be the set of the $(t+1)$ processes $p_{x}$ such that, at time $\tau$, SUSPICIONS $[x, k] \leq s k_{t+1}(\tau)$. We consider two cases.

1. $S_{k}=K(\tau)$. Then, taking $p_{i}$ as the "last" process of $S_{k}$ such that $\operatorname{SUSPICIONS}[i, k]=s k_{t+1}(\tau)$ proves the lemma.

2. $S_{k} \neq K(\tau)$. In that case, let us take $p_{i}$ as a process in $S_{k} \backslash K(\tau)$. As $p_{i} \notin K(\tau)$, it follows from the definition of $K(\tau)$ that $S U S P I C I O N S[i, k] \geq s k_{t+1}(\tau)$, and the lemma follows.

$\square_{\text {Lemma } 3}$

Notation 3 Let $M_{x}=\max \left(\left\{M_{x}(\tau)_{\tau \geq 0}\right\}\right)$. If there is no such value $\left(M_{x}(\tau)\right.$ grows forever according to $\left.\tau\right)$, let $M_{x}=+\infty$. Let $B$ be the set of processes $p_{x}$ such that $M_{x}$ is bounded.

Lemma $4 A W B \Rightarrow(B \neq \emptyset)$.

Proof We consider two cases.

- Case $f=t$. Let us first observe that, for no process $p_{k}$ updates $S U S P I C I O N S[k, k]$. Let us consider a time $\tau$ after which the $t$ processes have crashed. Let $p_{j}$ be any of these processes. It follows from Lemma 1 that, after $\tau, p_{j}$ never updates $S U S P I C I O N S[j, k]$. Consequently, for any correct process $p_{k}$, there are $(t+1)$ entries $S U S P I C I O N S[1 . . n, k]$ that are no longer modified after $\tau$. It follows that $B$ is not empty.

- Case $f<t$. Let $p_{k}$ be a process that satisfies $A W B_{1}$. We show that $M_{k}$ is bounded. Due to Lemma 3, at any time $\tau$, there is a process $p_{j(\tau)} \in S_{k}$ such that we have $\operatorname{SUSPICIONS}[j(\tau), k](\tau) \geq s k_{t+1}(\tau)$. (where SUSPICIONS $[j(\tau), k](\tau)$ denotes the value of the corresponding variable at time $\tau)$. It follows that $M_{k}(\tau)$ is upper bounded by $(t+1) \times S U S P I C I O N S[j(\tau), k](\tau)$. So, the proof amounts to show that, after some time, for any $j \in S_{k}, S U S P I C I O N S[j, k]$ remains bounded. Let us consider any process $p_{j} \in S_{k}$ after the time at which the $f$ faulty processes have crashed. There are three cases.

1. $p_{j}=p_{k}$. In this case $S U S P I C I O N S[j, k]=1$ permanently.

2. $p_{j}$ is a faulty process of $S_{k}$. SUSPICIONS $[j, k]$ is then bounded due to Lemma 1 .

3. $p_{j}$ is a process of $S_{k}$ that is one of the $(t-f)$ correct processes involved in the assumption $A W B_{2}$. $S U S P I C I O N S[j, k]$ is then bounded due to Lemma 2.

$\square_{\text {Lemma } 4}$

Lemma 5 There is a time after which any invocation of the primitive leader () issued by a process, returns the identity of a process of $B$.

Proof The lemma follows from the lines 2-6 and the fact that $B$ is not empty (Lemma 4).

$\square$ Lemma 5

Notation 4 Let $\left(M_{a}, a\right)=\operatorname{lex} \_\min \left(\left\{\left(M_{x}, x\right) \mid p_{x} \in B\right\}\right)$.

Lemma 6 There is a single process $p_{a}$ and it is a correct process.

Proof Let us first observe that $B \neq \emptyset$ (Lemma 4). Moreover, as no two processes have the same identity, there is a single process $p_{a}$ such that $\left(M_{a}, a\right)=$ lex_min $\left(\left\{\left(M_{x}, x\right) \mid p_{x} \in B\right\}\right.$. So, the proof of the lemma consists in showing that $p_{a}$ is a correct process.

Let assume by contradiction that $p_{a}$ is a faulty process. This means that there is a time $\tau_{a 1}$ after which $p_{a}$ does no longer update PROGRESS $[a]$. As $\left(M_{a}, a\right)=\operatorname{lex} \_\min \left(\left\{\left(M_{x}, x\right) \mid p_{x} \in B\right\}\right)$, it follows that, after some time $\tau_{a 2}, p_{a}$ is permanently considered leader by all the processes $p_{i}$, and consequently, each time its timer expires, $p_{i}$ is such that $k=a$ (line 16). Let $\tau \geq \max \left(\tau_{a 1}, \tau_{a 2}\right)$. After $\tau$, there is at least one correct process $p_{i}$ that, each time it executes line 19 is such that $(k=a) \wedge\left(i \in\right.$ witness $\left._{\_} k_{i}\right)$ (that correct process is not necessarily always the same). Moreover, as PROGRESS $[a]$ remains constant, we then always have PROGRESS $[a]=$ last $_{i}[k]$. Consequently, infinitely often one of the $(t+1)$ smallest entries of SUSPICIONS $[1 . . n, a]$ is increased, contradicting the fact that $M_{a}$ is bounded.

$\square_{\text {Lemma } 6}$ 
Theorem 1 There is a time after which all the invocations leader() return the identity of the same correct process.

Proof It follows from Lemma 5 that, after some finite time, all the leader() invocations return the identity of a process of $B$. It follows from lines 2-6 that this identity is the identity $a$ defined in Notation 4 . Lemma 6 has shown that $p_{a}$ is a correct process.

$\square_{\text {Theorem } 1}$

Theorem 2 The protocol is write-optimal (i.e., after some time a single process writes the shared memory). Moreover, be the execution finite or infinite, all variables, but one entry of PROGRESS, are bounded.

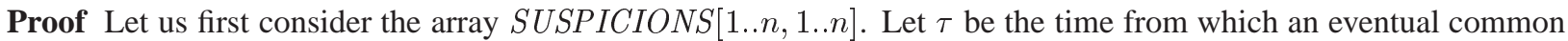
leader $p_{\ell}$ is elected. Due to Theorem 1 such a time $\tau$ does exist. After time $\tau$ we have the following.

- As, after $\tau$, any invocation of leader() at line 16 by a process $p_{i}$ returns always $\ell$, we conclude that $\forall i, \forall j \neq \ell$, $S U S P I C I O N S[i, j]$ is never updated after $\tau$ (line 24 ).

- Let $\tau^{\prime}$ be the time from which we have $M_{\ell}\left(\tau^{\prime}\right)=M_{\ell}$, and $\tau^{\prime \prime}=\max \left(\tau, \tau^{\prime}\right)$. We now show that no process $p_{i}$ increases SUSPICIONS $[i, \ell]$ more than once after $\tau^{\prime \prime}$, which implies that eventually SUSPICIONS $[i, \ell]$ is not updated anymore.

Let us consider process $p_{i}$ that evaluates the predicate of line 19 after $\tau^{\prime \prime}$. We then have $k=\ell$.

- The predicate is true. In that case, the sub-predicate $i \in$ witness $k_{i}$ is also true. This means that if $p_{i}$ increased SUSPICIONS $[i, \ell]$, either $M_{\ell}$ would be increased or not. The first case contradicts the definition of $M_{\ell}$ (namely, $M_{\ell}=\max \left(\left\{M_{\ell}(\tau)_{\tau>0}\right\}\right)$ ). On the other hand, if $M_{\ell}$ is not increased, then this implies that $p_{i}$ stops being a witness of $p_{\ell}$. Therefore, in all further evaluations of line 19 by $p_{i}$ the predicate will be false.

- The predicate is false. In that case, it follows directly from the text of the protocol that the shared variable $S U S P I C I O N S[i, \ell]$ is not updated.

Let us now consider any shared variable PROGRESS[i], $1 \leq i \neq \ell \leq n$. This variable is updated at line 11. After $p_{\ell}$ has been elected, the predicate $\operatorname{leader}()=i$ is always false. Moreover, as we have seen previously, there is a time $\tau^{\prime}$ after which no variable $S U S P I C I O N S[x, y]$ is updated. It follows that, after $\tau^{\prime}$, the predicate susp_count $_{i} \neq$ prev_susp_count $_{i}$ is always false. It follows that, there is a time after which no PROGRESS[i] variable, $1 \leq i \neq \ell \leq n$, can be updated; which concludes the proof of the theorem.

$\square$ Theorem 2

Corollary 1 Be the execution finite or infinite, all the timeout values remain bounded.

Proof The corollary is an immediate consequence of Theorem 2 and line 28 of Figure 2.

$\square$ Corollary 1

On the process that is elected The proof of the protocol relies on the assumption $A W B_{1}$ to guarantee that at least one correct process can be elected (i.e., the set $B$ is not empty, -Lemma 4-, and its smallest pair $\left(M_{a}, a\right)$ is such that $p_{a}$ is a correct process -Lemma 6-). This does not mean that the elected process is a process that satisfies the assumption $A W B_{1}$. There are cases where it can be another process.

To see when this can happen, let us consider two correct processes $p_{i}$ and $p_{j}$ such that $p_{i}$ does not satisfy $A W B_{2}$ (its timer is never well-behaved) and $p_{j}$ does not satisfy $A W B_{1}$ (it never behaves synchronously). (A re-reading of the statement of Lemma 2 will make the following description easier to understand.) Despite the fact that (1) $p_{i}$ is not synchronous with respect to a process that satisfies $A W B_{1}$, and can consequently suspects these processes infinitely often, and (2) $p_{j}$ is not synchronous with respect to a process that satisfy $A W B_{2}$ (and can consequently be suspected infinitely often by such processes), it is still possible that $p_{i}$ and $p_{j}$ behave synchronously one with respect to the other in such a way that $p_{i}$ never suspects $p_{j}$. If this happens $S U S P I C I O N S[i, j]$ remains bounded, and it is possible that the value $M_{j}$ not only remains bounded, but becomes the smallest value in the set $B$. It this occurs, $p_{j}$ is elected as the common leader.

Of course, there are runs in which the previous scenario does not occur. That is why the protocol has to rely on $A W B_{1}$ in order to guarantee that the set $B$ be never empty.

PI ${ }^{\circ} 1842$ 


\subsection{Optimality Results}

Let $\mathcal{A}$ be a protocol that implements $\Omega$ in $\mathcal{A S}_{n, t}[A W B]$. We have the following lower bounds.

Lemma 7 Let $R$ be any run of $\mathcal{A}$ with less than $t$ faulty processes and let $p_{\ell}$ be the leader chosen in $R$. Then $p_{\ell}$ must write forever in the shared memory in $R$.

Proof Assume, by way of contradiction, that $p_{\ell}$ stops writing in the shared memory in run $R$ at time $\tau$. Consider another run $R^{\prime}$ of $\mathcal{A}$ in which all processes behave like in $R$ except $p_{\ell}$, which behaves exactly like in $R$ until time $\tau+1$, and crashes at that time. Since at most $t$ processes crash in $R^{\prime}$, by definition of $\mathcal{A}$, eventually a leader must be elected. In fact, in $R^{\prime}$ all the processes except $p_{\ell}$ behave exactly like in $R$ and elect $p_{\ell}$ as their (permanent) leader. These processes cannot distinguish $R^{\prime}$ from $R$ and cannot detect the crash of $p_{\ell}$. Hence, in $R^{\prime}$ protocol $\mathcal{A}$ does not satisfy the Eventual Leadership property of $\Omega$, which is a contradiction. Therefore, $p_{\ell}$ cannot stop writing in the shared memory.

$\square_{\text {Lemma } 7}$

Lemma 8 Let $R$ be any run of $\mathcal{A}$ with less than $t$ faulty processes and let $p_{\ell}$ be the leader chosen in $R$. Then every correct process $p_{i}, i \neq \ell$, must read forever from the shared memory in $R$.

Proof Assume, by way of contradiction, that a correct process $p_{i}$ stops reading from the shared memory in run $R$ at time $\tau$. Let $\tau^{\prime}$ be the time at which $p_{i}$ chooses permanently $p_{\ell}$ as leader. Consider another run $R^{\prime}$ of $\mathcal{A}$ in which $p_{\ell}$ behaves exactly like in $R$ until time $\max \left(\tau, \tau^{\prime}\right)+1$, and crashes at that time. Since at most $t$ processes crash in $R^{\prime}$, by definition of $\mathcal{A}$, a leader must be eventually elected. In $R^{\prime}$, we make $p_{i}$ to behave exactly like in $R$. As it stopped reading the shared memory at time $\tau, p_{i}$ cannot distinguish $R^{\prime}$ from $R$ and cannot detect the crash of $p_{\ell}$. Hence in $R^{\prime}, p_{i}$ elects $p_{\ell}$ as its (permanent) leader at time $\tau^{\prime}$. Hence, in $R^{\prime}$ protocol $\mathcal{A}$ does not satisfy the Eventual Leadership property of $\Omega$, which is a contradiction. Therefore, $p_{i}$ cannot stop reading from the shared memory. $\quad \square_{\text {Lemma } 8}$

The following theorem follows immediately from the previous lemmas.

Theorem 3 The protocol described in Figure 2 is optimal with respect to the number of processes that have to write the shared memory. It is quasi-optimal with respect to the number of processes that have to read the shared memory.

The "quasi-optimality" comes from the fact that the protocol described in Figure 2 requires that each process (including the leader) reads forever the shared memory (all the processes have to read the array SUSPICIONS $[1 . . n, 1 . . n]$ ).

\section{A $t$-resilient protocol for $\mathcal{A S}_{n, t}[A W B]$ with bounded variables only}

\subsection{A Lower Bound Result}

This section shows that any protocol that implements an eventual leader service $\Omega$ in $\mathcal{A S}_{n, t}[A W B]$ with only bounded memory requires all correct processes to read and write the shared memory forever. As we will see, it follows from this lower bound that the protocol described in Figure 4 is optimal with respect to this criterion.

Let $\mathcal{A}$ be a protocol that implements $\Omega$ in $\mathcal{A S}_{n, t}[A W B]$ such that, in every run $R$ of $\mathcal{A}$, the number of shared memory bits used is bounded by a value $S_{R}$ (which may depend on the run). This means that in any run there is time after which no new memory positions are used, and each memory position has bounded number of bits.

Theorem 4 The protocol $\mathcal{A}$ has runs in which at least $t+1$ processes write forever in the shared memory.

Proof To prove the claim we construct a run $R$ of $\mathcal{A}$ such that:

1. $R$ is fault free,

2. Process $p_{1}$ is synchronous while the rest of processes are asynchronous, and

3. There is an infinite sequence of times $\tau_{0}<\tau_{1}<\tau_{2}<\ldots$ such that, $\forall i>0$, in the interval $\left(\tau_{i-1}, \tau_{i}\right]$ some process changes its leader or at least $t+1$ processes write in the shared memory. 
Clearly, since a leader must be eventually elected in $R$ and the number of processes is finite, due to Item 3 , there is a set of at least $t+1$ processes that write in the shared memory forever.

For simplicity, let us define $\tau_{0}=0$. This will be the base case. Then, for $i>0$ let us assume $R$ is already constructed up to time $\tau_{i-1}$. We construct now interval $\left(\tau_{i-1}, \tau_{i}\right]$. This interval is constructed differently depending on which of the following two cases occurs.

- If at time $\tau_{i-1}$ the leader of some process $p_{j}$ is an asynchronous process $p_{k}$ (i.e., $k \neq 1$ ), we first consider a run $R_{i}$ that behaves exactly like $R$ up to time $\tau_{i-1}$. Then, after that time all processes advance synchronously (e.g., one step per time unit), except $p_{k}$ which crashes at time $\tau_{i-1}+1$. By Eventual Leadership, there is a time $\tau>\tau_{i-1}$ in $R_{i}$ at which no process considers $p_{k}$ as its leader. Then, let us define $\tau_{i}=\tau+1$ and make $R$ to behave in the interval $\left(\tau_{i-1}, \tau_{i}\right]$ as follows. All processes except $p_{k}$ behave in this interval exactly like in the interval $\left(\tau_{i-1}, \tau_{i}\right]$ of $R_{i}$. Process $p_{k}$ does not crash, but is stopped at time $\tau_{i-1}+1$ and does not execute any step until the end of the interval. This behavior is possible since $p_{k}$ is asynchronous. Then, we have that in the interval $\left(\tau_{i-1}, \tau_{i}\right]$ some process changed its leader. This ends the first case.

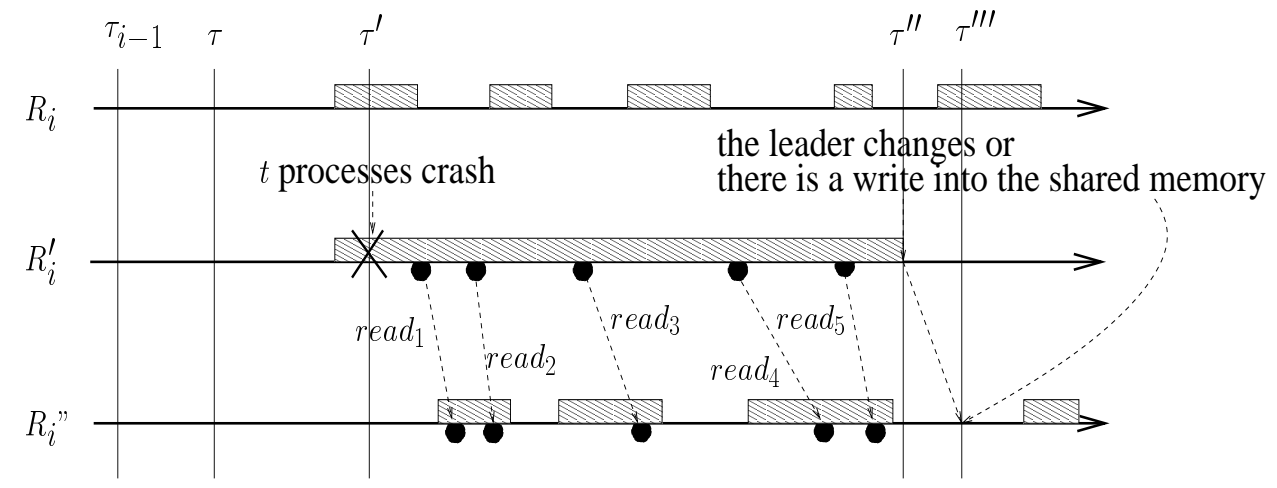

Figure 3: Illustrating the runs $R_{i}, R_{i}^{\prime}$ and $R_{i}^{\prime \prime}$

- The second case occurs when at time $\tau_{i-1}$ in $R$ the leader of all processes is the synchronous process $p_{1}$. As before we now consider an auxiliary run $R_{i}$ that behaves exactly like $R$ up to time $\tau_{i-1}$. After that time all processes advance synchronously (e.g., one step per time unit) in $R_{i}$. If some process $p_{j}$ changes its leader in $R_{i}$ at some time $\tau>\tau_{i-1}$, then we define $\tau_{i}=\tau+1$ and make the interval $\left(\tau_{i-1}, \tau_{i}\right]$ of $R$ behave exactly as interval $\left(\tau_{i-1}, \tau_{i}\right]$ of $R_{i}$.

Otherwise, if no process changes its leader in $R_{i}$ after $\tau_{i-1}$, we have from Lemma 7 that $p_{1}$ writes in the shared memory forever. Let us assume by way of contradiction that there is a time $\tau>\tau_{i-1}$ after which at most $t-1$ other processes write forever in the shared memory in $R_{i}$. Since the shared memory is bounded, some state (understood as the value of all its bits) $S$ of the shared memory must occur infinitely often in $R_{i}$ after $\tau$. (First line in Figure 3 where the state $S$ is described with an area with stripes.)

Let us consider now a run $R_{i}^{\prime}$ which behaves exactly like $R_{i}$ up to time $\tau^{\prime}>\tau$ at which the shared memory is in state $S$ (second line in Figure 3). Then, at that time the (up to $t$ ) processes that were writing in the shared memory (including $p_{1}$ ) crash in $R_{i}^{\prime}$. The rest of the processes advance synchronously (and hence the $A W B_{1}$ assumption holds in $R_{i}^{\prime}$ ) until the smallest time $\tau^{\prime \prime}>\tau^{\prime}$ at which some process changes its leader or some process writes in the shared memory. This must eventually occur by Eventual Leadership, since the leader of all the processes at time $\tau^{\prime}$ has crashed in $R_{i}^{\prime}$. Note that in the interval $\left(\tau^{\prime}, \tau^{\prime \prime}\right)$ all read operations find the shared memory in state $S$.

Consider now another run $R_{i}^{\prime \prime}$ in which the up to $t$ processes (including $p_{1}$ ) that write forever in $R_{i}$ behave like they do in that run, while the rest of processes (let us denote this set of processes by $L$ ) behave like in $R_{i}$ up to time $\tau^{\prime}$ (last line in Figure 3.) After $\tau^{\prime}$, the processes in $L$ are delayed (note that they are all asynchronous) so that every time they read form the shared memory they find it in state $S$ (see Figure 3). From the behavior of the processes in $L$ in run $R_{i}^{\prime}$ and the fact that they cannot distinguish run $R_{i}^{\prime \prime}$ from run $R_{i}^{\prime}$, we have that there is a time $\tau^{\prime \prime \prime}>\tau^{\prime}$ at which some process in $L$ changes its leader or writes in the shared memory in run $R_{i}^{\prime \prime}$. Then, we define $\tau_{i}=\tau^{\prime \prime \prime}+1$ and make interval $\left(\tau_{i-1}, \tau_{i}\right]$ of $R$ behave exactly like that interval in $R_{i}^{\prime \prime}$. 
Figure 3 summarizes the previous reasoning. In the first run $R_{i}$, after $\tau$, only $t$ processes write forever. The same state $S$ (depicted by the area with stripes) occurs repeatedly forever. In the run $R_{i}^{\prime}$, these $t$ processes crash in state $S$ (they crash at the time marked with a cross). The read operations from the other processes are indicated with black dots. In the run $R_{i}^{\prime \prime}$, the same processes as in $R_{i}^{\prime}$ read while the system in the state $S$.

$\square_{\text {Theorem } 4}$

\subsection{A protocol with only bounded variables}

Principles and description As already indicated, we are interested here in a protocol whose variables are all bounded. To attain this goal, we use a hand-shaking mechanism. More precisely, we replace the shared array PROGRESS $[1 . . n]$ and all the local arrays last $_{i}[1 . . n], 1 \leq i \leq n$, by two shared matrices of 1 WMR boolean values, denoted PROGRESS[1..n, 1..n] and LAST[1..n, 1..n].


$L A S T[i, k]$ are used by these processes to send signals to each other. More precisely, to signal $p_{k}$ that it is alive, $p_{i}$ sets PROGRESS $[i, k]$ equal to $\neg L A S T[i, k]$. In the other direction, $p_{k}$ indicates that it has seen this "signal" by cancelling it, namely, it resets $\operatorname{LAST}[i, k]$ equal to PROGRESS $[i, k]$. So, $p_{i}$ writes PROGRESS $[i, k]$ and $L A S T[k, i]$, while $p_{k}$ reads them. It follows from the essence of the hand-shaking mechanism that both $p_{i}$ and $p_{k}$ have to write shared variables, but as shown by Corollary 2 , this is the price that has to be paid to have bounded shared variables.

Using this simple technique, we obtain the protocol described in Figure 4. Let us recall that $p_{i}$ is the owner of PROGRESS $[i, k]$ and $L A S T[k, i], 1 \leq k \leq n$, i.e., it is the only process that can write them. So, $p_{i}$ manages two additional local arrays progress $s_{i}[1 . . n]$ and last $_{i}[1 . . n]$, such that progress $i[k]$ is a local copy of PROGRESS $[i, k]$, and last $_{i}[k]$ is a local copy of $L A S T[k, i]$. (As in the first protocol, this allows saving shared memory accesses.)

In order to capture easily the parts that are new or modified with respect to the previous protocol, the line number of the new statements are suffixed with the letter $\mathrm{R}$ (so the line 11 of the previous protocol is replaced by six new lines 11.R1-11.R6, while each of the lines 20, 21 and 22 is replaced by a single line). This allows a better understanding of the common principles on which both protocols rely.

Proof of the protocol The statement of the lemmas 1-6, and Theorem 1 are still valid when the shared array PROGRESS $[1 . . n]$ is replaced by the shared matrices PROGRESS $[1 . . n, 1 . . n]$ and $L A S T[1 . . n, 1 . . n]$. As far as their proofs are concerned, the proofs of Lemma 1, Lemma 3, Lemma 4, Lemma 5, Lemma 6, and Theorem 1 are nearly verbatim the same.

The proofs of Lemma 2 has to be slightly modified to suit the new context. Basically, it differs from its counterparts of Section 3.4 in the way it establishes the property that, after some time, no correct process $p_{i}$ misses an "alive" signal from a process that satisfies the assumption $A W B_{1}$. (More specifically, the sentence "there is a time after which PROGRESS $[k]$ does no longer increase" has to be replaced by the sentence "there is a time after which PROGRESS $[k, i]$ remains forever equal to $L A S T[k, i]$ ".) As it is very close to its counterpart (and tedious), we don't detail this proof. (According to the usual sentence, "this proof is left as an exercise to the reader".)

A reasoning similar to the one in the proof of Theorem 2 shows that each variable SUSPICIONS $[j, k], 1 \leq j, k \leq$ $n$, is bounded. Combined with the fact that the variables PROGRESS $[j, k]$ and $L A S T[j, k]$ are boolean, we obtain the following theorem.

Theorem 5 All the variables used in the protocol described in Figure 4 are bounded.

Concerning the variables that are updated, we have the following theorem.

Theorem 6 Let $p_{\ell}$ be the process elected as the eventual common leader in the protocol described in Figure 4. There is a set of $t$ processes $p_{i}, i \neq \ell$, such that eventually the only variables that may be written are PROGRESS[ $[, i]$ (written by $p_{\ell}$ ) and $L A S T[\ell, i]$ (written by $p_{i}$ ).

Proof The proof that (1) there is a time after which the variables $\operatorname{SUSPICIONS}[j, k], 1 \leq j, k \leq n$, are no longer written, and the proof that (2) there is a time after which PROGRESS[x,j], $\leq x, j \leq n, x \neq \ell$, is no longer written, are the same as the proof done in Theorem 2. Let us now consider any variable $L A S T[x, y], x \neq \ell$. As, after $p_{\ell}$ has been elected, no correct process $p_{x}, x \neq \ell$, updates PROGRESS[x,y] (line 11.R3), it follows that there is a time after which the predicate $\operatorname{LAST}[x, y]=\operatorname{PROGRESS}[x, y]$ remains forever true for $1 \leq x, y \leq n$ and $x \neq \ell$. 


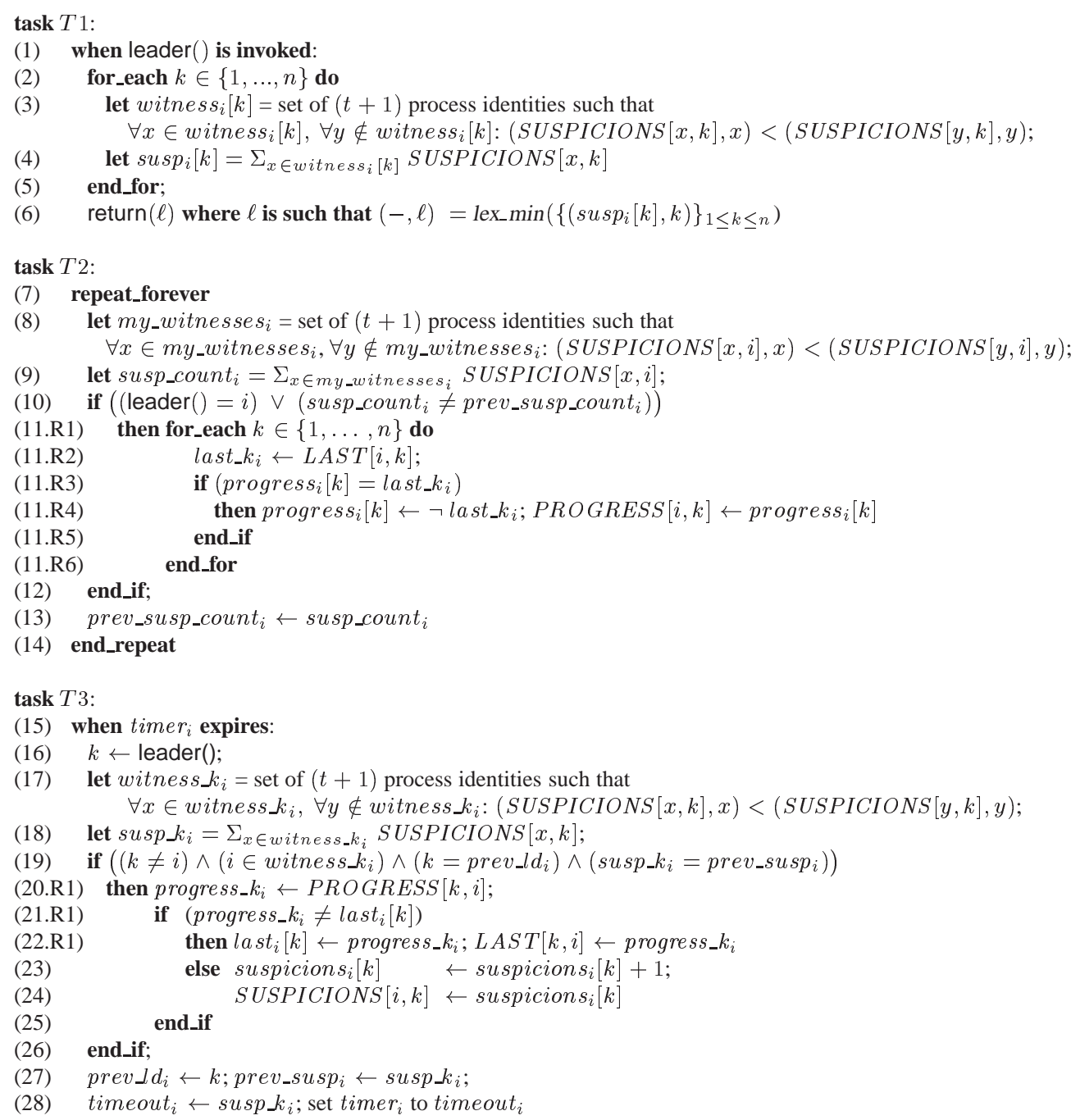

Figure 4: $t$-resilient eventual leader election with all variables bounded (code for $p_{i}$ )

Consequently, after a finite time, the test of line 21.R1 is always false for $p_{x}, x \neq \ell$, and $\operatorname{LAST}[x, y]$ is no longer written.

The fact that SUSPICIONS $[j, k], 1 \leq j, k \leq n$, eventually never changes implies that the set of witnesses of $p_{\ell}$ will eventually stabilize. A process $p_{x}$ that is not in this set of stable witnesses of $p_{\ell}$ eventually stops writing $\operatorname{LAST}[\ell, x]$, because the predicate at line 19 is always false. Once this has happened, $p_{\ell}$ will eventually set PROGRESS $[\ell, x]=\neg L A S T[\ell, x]$ (line 11.R4). After that, the predicate at line 11.R3 remains forever false and $P R O G R E S S[\ell, x]$ is no longer written. Additionally, note that $p_{\ell}$ is always witness of itself, since initially $S U S P I C I O N S[\ell, \ell]=$

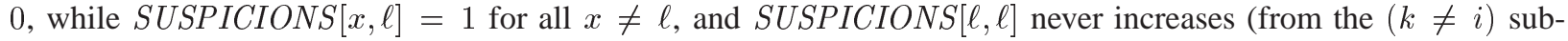
predicate at line 19). Note as well that $L A S T[\ell, \ell]$ is never modified (also from the $(k \neq i)$ sub-predicate at line 19), and hence $P R O G R E S S[\ell, \ell]$ eventually stops being written.

Hence, only the variables PROGRESS $[\ell, x]$ and $L A S T[\ell, x]$ for the set of $t$ processes $p_{x}, x \neq \ell$, that are stable witnesses of $p_{\ell}$ are written forever.

Finally, the next corollary follows directly from the above theorem and Theorem 4. 
Corollary 2 The protocol described in Figure 4 is optimal with respect to the number of processes that have to write the shared memory.

\section{Conclusion}

This paper has addressed the problem of electing an eventual leader in an asynchronous shared memory system. It has three main contributions.

- The first contribution is the statement of an assumption (a property denoted $A W B$ ) that allows electing a leader in the shared memory asynchronous systems that satisfy that assumption. This assumption requires that after some time (1) there is a process whose write accesses to some shared variables are timely, and (2) the other processes have asymptotically well-behaved timers. The notion of asymptotically well-behaved timer is weaker than the usual timer notion (where the timer durations have to monotonically increase when the values to which they are set increase). This means that $A W B$ is a particularly weak assumption.

- The second contribution is the design of two protocols that elect an eventual leader in any asynchronous shared memory system that satisfies the assumption $A W B$. In addition of being $t$-resilient (where $t$ is the maximum number of processes allowed to crash), and being based only on one-writer/multi-readers atomic shared variables, these protocols enjoy noteworthy properties. The first protocol guarantees that (1) there is a (finite) time after which a single process writes forever the shared memory, and (2) all but one shared variables have a bounded domain. The second protocol uses (1) a bounded memory but (2) requires that each process forever writes the shared memory.

- The third contribution shows that the previous tradeoff (bounded/unbounded memory vs number of processes that have to write) is inherent to the leader election problem in asynchronous shared memory systems equipped with $A W B$. It follows that both protocols are optimal, the first with respect to the number of processes that have to forever write the shared memory, the second with respect to the boundedness of the memory.

Several questions remain open. One concerns the first protocol. Is it possible to design a leader protocol in which there is a time after which the eventual leader is not required to read the shared memory? Another question is the following: is the second protocol optimal with respect to the size of the control information (bit arrays) it uses to have a bounded memory implementation?

\section{References}

[1] Abraham I., Chockler G.V., Keidar I. and Malkhi D., Byzantine Disk Paxos, Optimal Resilience with Byzantine Shared Memory. Proc. 23th ACM Symposium on Principles of Distributed Computing (PODC'04), ACM Press, pp. 226-235, 2004.

[2] Aguilera M.K., Delporte-Gallet C., Fauconnier H. and Toueg S., On Implementing Omega with Weak Reliability and Synchrony Assumptions. Proc. 22th ACM Symposium on Principles of Distributed Computing (PODC'03), ACM Press, pp. 306-314, 2003.

[3] Aguilera M.K., Delporte-Gallet C., Fauconnier H. and Toueg S., Communication-Efficient Leader Election and Consensus with Limited Link Synchrony. Proc. 23th ACM Symposium on Principles of Distributed Computing (PODC'04), ACM Press, pp. 328-337, 2004.

[4] Aguilera M.K., Englert B. and Gafni E., On Using Network Attached Disks as Shared Memory. Proc. 21th ACM Symposium on Principles of Distributed Computing (PODC'03), ACM Press, pp. 315-324, 2003.

[5] Chandra T.D. and Toueg S., Unreliable Failure Detectors for Reliable Distributed Systems. Journal of the ACM, 43(2):225267, 1996.

[6] Chandra T., Hadzilacos V. and Toueg S., The Weakest Failure Detector for Solving Consensus. Journal of the ACM, 43(4):685$722,1996$.

[7] Dwork C., Lynch N. and Stockmeyer L., Consensus in the Presence of Partial Synchrony. Journal of the ACM, 35(2):288-323, 1988. 
[8] Fernández A., Jiménez E. and Raynal M., Electing an Eventual Leader in an Asynchronous Shared Memory System. Proc. 37th Int'l IEEE Conference on Dependable Systems and Networks (DSN'07), IEEE Computer Society Press, Edinburgh (UK), June 2007.

[9] Gafni E. and Lamport L., Disk Paxos. Distributed Computing, 16(1):1-20, 2003.

[10] Gibson G.A. et al., A Cost-effective High-bandwidth Storage Architecture. Proc. 8th Int'l Conference on Architectural Support for Programming Languages and Operating Systems (ASPLOS'98), ACM Press, pp. 92-103, 1998.

[11] Guerraoui R., Kapalka M. and Kouznetsov P., The Weakest failure Detectors to Boost Obstruction-Freedom. Proc. 20th Symposium on Distributed Computing (DISC’06), Springer-Verlag LNCS \#4167, pp. 376-390, 2006.

[12] Guerraoui R. and Raynal M., The Information Structure of Indulgent Consensus. IEEE Transactions on Computers, 53(4):453-466, 2004.

[13] Guerraoui R. and Raynal M., A Leader Election Protocol for Eventually Synchronous Shared Memory Systems. 4th Int'l IEEE Workshop on Software Technologies for Future Embedded and Ubiquitous Systems (SEUS'O6), IEEE Computer Society Press, pp. 75-80, 2006.

[14] Guerraoui R. and Raynal M., The Alpha of Asynchronous Consensus. The Computer Journal, 50(1):53-67, 2007.

[15] Herlihy M.P., Wait-Free Synchronization. ACM Transactions on programming Languages and Systems, 11(1):124-149, 1991.

[16] Herlihy M.P., Luchangco V. and Moir M., Obstruction-free Synchronization: Double-ended Queues as an Example. Proc. 23th IEEE Int'l Conference on Distributed Computing Systems (ICDCS’03), pp. 522-529, 2003.

[17] Herlihy M.P., Luchangco V., Moir M. and Scherer III W.N., Software Transactional Memory for Dynamic Sized Data Structure. Proc. 21th ACM Symp. on Principles of Distributed Computing (PODC'03), pp. 92-101, 2003.

[18] Herlihy M.P. and Wing J.M., Linearizability: a Correctness Condition for Concurrent Objects. ACM Transactions on Programming Languages and Systems, 12(3):463-492, 1990.

[19] Lamport L., The Part-Time Parliament. ACM Transactions on Computer Systems, 16(2):133-169, 1998. (The first version of Paxos appeared a a DEC Tech Report in 1989.)

[20] Larrea M., Fernández A. and Arévalo S., Optimal Implementation of the Weakest Failure Detector for Solving Consensus. Proc. 19th Symposium on Resilient Distributed Systems (SRDS'00), IEEE Computer Society Press, pp. 52-60, 2000.

[21] Lee E.K. and Thekkath C., Petal: Distributed Virtual Disks. Proc. 7th Int'l Conference on Architectural Support for Programming Languages and Operating Systems (ASPLOS'96), ACM Press, pp. 84-92, 1996.

[22] Lo W.-K. and Hadzilacos V., Using failure Detectors to solve Consensus in Asynchronous Shared Memory Systems. Proc. 8th Int'l Workshop on Distributed Computing (WDAG'94), Springer Verlag LNCS \#857, pp. 280-295, 1994.

[23] Malkhi D., Oprea F. and Zhou L., $\Omega$ Meets Paxos: Leader Election and Stability without Eventual Timley Links. Proc. 19th Int'l Symposium on DIStributed Computing (DISC'05), Springer Verlag LNCS \#3724, pp. 199-213, 2005.

[24] Mostefaoui A., Mourgaya E., and Raynal M., Asynchronous Implementation of Failure Detectors. Proc. Int'l IEEE Conference on Dependable Systems and Networks (DSN'03), IEEE Society Press, pp. 351-360, 2003.

[25] Mostefaoui A. and Raynal M., Leader-Based Consensus. Parallel Processing Letters, 11(1):95-107, 2001.

[26] Mostéfaoui A., Mourgaya E., Raynal M. and Travers C., Time-free Assumption to Implement Eventual Leadership. Parallel Processing letters, 16(2):189-208, 2006.

[27] Mostéfaoui A., Raynal M. and Travers C., Time-free and Timeliness Assumptions can be Combined to Get Eventual Leadership. IEEE Transactions on Parallel and Distributed Systems, 17(7):656-666, 2006.

[28] Powell D., Failure Mode Assumptions and Assumption Coverage. Proc. of the 22nd Int'l Symposium on Fault-Tolerant Computing (FTCS-22), Boston, MA, pp.386-395, 1992.

[29] Raynal M., A Short Introduction to Failure Detectors for Asynchronous Distributed Systems. ACM SIGACT News, Distributed Computing Column, 36(1):53-70, 2005. 